\title{
Solamargine derived from Solanum nigrum induces apoptosis of human cholangiocarcinoma QBC939 cells
}

\author{
XIUHUA ZHANG ${ }^{1,2^{*}}$, ZHANPENG YAN $^{1,3^{*}}$, TINGTING XU $^{1,3}$, ZHENTAO AN $^{1}$, \\ WANZHEN CHEN ${ }^{1}$, XIAOSONG WANG ${ }^{2}$, MENGMENG HUANG ${ }^{3}$ and FANGSHI ZHU ${ }^{1,3}$
}

\begin{abstract}
${ }^{1}$ Clinical Research Department of Chinese and Western Medicine, Affiliated Hospital of Integrated Traditional Chinese and Western Medicine, Nanjing University of Chinese Medicine, Nanjing, Jiangsu 210028; ${ }^{2}$ Department of Gastroenterology, Second Affiliated Hospital of Nanjing Medical University, Nanjing, Jiangsu 210011; ${ }^{3}$ Clinical Research Department of

Chinese and Western Medicine, Jiangsu Province Institute of Traditional

Chinese Medicine, Nanjing, Jiangsu 210028, P.R. China
\end{abstract}

Received July 14, 2016; Accepted November 7, 2017

DOI: $10.3892 / \mathrm{ol} .2018 .8171$

\begin{abstract}
Solamargine, an activeingredient of Solanumnigrum, has been previously revealed to inhibit the proliferation of cancer cells. However, the effect of solamargine on human cholangiocarcinoma cells and the underlying molecular mechanism remain unknown. In the present study, the molecular mechanism underlying the anti-cancer effect of solamargine was assessed in human cholangiocarcinoma QBC939 cells. The results of the present study revealed that solamargine inhibited the viability of QBC939 cells in a dose-dependent manner. Furthermore, solamargine significantly induced the apoptosis of QBC939 cells and altered the mitochondrial membrane potential of cells. Quantitative polymerase chain reaction analysis revealed that solamargine decreased the mRNA level of B-cell lymphoma-2 (Bcl-2), Bcl-extra-large and $\mathrm{X}$-linked inhibitor of apoptosis protein but increased the mRNA level of Bcl-2-associated X protein (Bax). In addition, western blot analysis demonstrated that solamargine inhibited the protein expression of Bcl-2 and poly ADP ribose polymerase (PARP), and promoted the protein expression of Bax, cleaved PARP, caspase 3, cleaved caspase 3 and caspase 7. Therefore, the results of the present study revealed that solamargine may induce apoptosis via the mitochondrial pathway and alter the level of apoptosis-associated proteins in human cholangiocarcinoma QBC939 cells. This in vitro
\end{abstract}

Correspondence to: Professor Fangshi Zhu or Mr. Zhanpeng Yan, Clinical Research Department of Chinese and Western Medicine, Jiangsu Province Institute of Traditional Chinese Medicine, 100 Shizi Street, Hongshan Road, Nanjing, Jiangsu 210028, P.R. China

E-mail: yzpsxxazz@126.com

E-mail: yanzhanpeng@126.com

*Contributed equally

Key words: solamargine, apoptosis, human cholangiocarcinoma study demonstrated that solamargine may be an effective chemotherapeutic agent against cholangiocarcinoma in clinical practice.

\section{Introduction}

Cholangiocarcinoma is a common primary biliary malignancy that originates from bile duct epithelial cells and has presented difficulties in diagnosis and treatment (1). Cholangiocarcinoma, including intrahepatic, perihilar, and distal cholangiocarcinoma, accounts for $\sim 10-15 \%$ of total hepatobiliary malignancies (2). The incidence of cholangiocarcinoma increased from 1832 cases in 2010 to 1964 cases in 2013 in England, and the majority of cholangiocarcinoma patients are $>60$ years old (3). Early diagnosis, operative treatment and chemotherapy for cholangiocarcinoma remain ineffective at treating cholangiocarcinoma (4). Cancer recurrence and metastasis have remained as the important mortality factors for patients with cholangiocarcinoma (5); therefore, the identification of an effective approach to treat human cholangiocarcinoma is required.

Solamargine is an alkaloid that is primarily derived from the Solanum nigrum plant. S. nigrum may exhibit heat-clearing and detoxifying effects, according to the theory of traditional Chinese medicine (6). Solamargine is an effective active ingredient of $S$. nigrum that may inhibit the proliferation and induce the apoptosis of multiple types of cancer cell, particularly human hepatocellular carcinoma cells (7-10). In addition, solamargine may enhance the susceptibility of human lung cancer and breast cancer to chemotherapeutic drugs (11-13). However, the effect of solamargine on human cholangiocarcinoma QBC939 cells and the underlying molecular mechanism remain unknown.

Apoptosis serves an important function in tumor formation and metastasis and is typically repressed in the tumor microenvironment $(14,15)$. Therefore, increasing the apoptosis induced by drugs is an effective method to inhibit cancer (16). In the present study, the effect of solamargine on the viability of cholangiocarcinoma QBC939 cells and associated molecular mechanisms was investigated and 
may provide experimental evidence of cholangiocarcinoma treated by solamargine.

\section{Materials and methods}

Materials. Solamargine (Fig. 1A), also known as (22R, $25 \mathrm{R})-3 \beta$-( $\beta$-D-Glucopy ranosyloxy) spirosol-5-ene or solasodine 3-glucoside, was purchased from Chendu Must Bio-Technology Co., Ltd. (Chendu, China) and dissolved in dimethyl sulfoxide (DMSO). The QBC939 cell line was obtained from Nanjing Chinese Medical University (Nanjing, China). RPMI 1640 medium, fetal bovine serum (FBS), $0.25 \%$ Trypsin-EDTA, penicillin and streptomycin were purchased from Gibco; Thermo Fisher Scientific, Inc. (Waltham, MA, USA). The mitochondrial membrane potential assay kit with JC-1, MTT and radioimmunoprecipitation assay (RIPA) protein lysis buffer were purchased from Beyotime Institute of Biotechnology (Haimen, China). The Annexin V-fluorescein isothiocyanate (FITC) apoptosis detection kit was purchased from BD Biosciences (Franklin Lakes, NJ, USA). The first cDNA synthesis kit for reverse transcription-quantitative polymerase chain reaction (RT-qPCR), the SYBR Green/ROX qPCR master mix and the protein ladder were obtained from Thermo Scientific, Inc. Caspase3 (catalog no. 9662), caspase7 (catalog no. 12827), X-linked inhibitor of apoptosis protein (XIAP) (catalog no. 2042), poly ADP ribose polymerase (PARP) (catalog no. 9542), B-cell lymphoma-2 (Bcl-2) (catalog no. 2876), Bcl-2-associated $\mathrm{X}$ protein (Bax) (catalog no. 2772) and $\beta$-actin (catalog no. 4970) antibodies were purchased from Cell Signaling Technology, Inc. (Danvers, MA, USA). Dylight 800-labeled goat anti-rabbit immunoglobulin $\mathrm{G}(\mathrm{H}+\mathrm{L})$ fluorescence antibody (catalog no. 072-07-15-06) was purchased from KPL, Inc. (Gaithersburg, MD, USA).

High-performance liquid chromatography (HPLC). $0.02 \mathrm{mg} / \mathrm{ml}$ solamargine is preparaed in $80 \%$ ethanol and detected by HPLC Agilent 1100 series (Agilent Technologies, Inc., Santa Clara, CA, USA). Chromatographic condition are displayed below. Chromatographic column: SinoChrom ODS-BP (C18), $5 \mu \mathrm{m}, 250 \times 4.6 \mathrm{~mm}$ (catalog no. 31110006, Dalian Elite Analytical Instruments Co., Ltd, Liaoning, China). Column temperature: $30^{\circ} \mathrm{C}$. Mobile phrase consists of acetonitrile and $0.1 \%$ ammonium hydroxide. The content of acetonitrile in gradient mobile phrase varies as below: From 25 to $45 \%$ in $0-20 \mathrm{~min}$; from 45 to $75 \%$ in $20-30 \mathrm{~min}$, flow rate is $1 \mathrm{ml} / \mathrm{min}$, detected at wavelength $203 \mathrm{~nm}$, sample loading volumn is $5 \mu 1$.

Cell culture. Human cholangiocarcinoma QBC939 cells were cultured in RPMI-1640 medium supplemented with $10 \%$ (v/v) FBS, $100 \mathrm{U} / \mathrm{ml}$ penicillin and $100 \mu \mathrm{g} / \mathrm{ml}$ streptomycin. Cells were maintained at $37^{\circ} \mathrm{C}$ in a humidified environment containing $5 \% \mathrm{CO}_{2}$. For all the experiments, cells were serum-starved and treated with solamargine for the specified times.

MTT assay and morphologic observation. QBC939 cells, in the period of logarithmic phase, were seeded in 96-well plate at a density of $1 \times 10^{4}$ cells/well in $100 \mu 1$ RPMI-1640 medium, in triplicate, and cultured at $37^{\circ} \mathrm{C}$ overnight in an atmosphere containing $5 \% \mathrm{CO}_{2}$. Cells were allowed to culture to $70 \%$ confluence/well and were treated with solamargine at the indicated concentration $(0,2,4,6,8,10,12$ and $14 \mu \mathrm{M})$ for $24 \mathrm{~h}$ at $37^{\circ} \mathrm{C}$. The morphology of QBC939 cells was observed by using inverted microscopy (magnification, x200) (Olympus Corporation, Tokyo, Japan). Subsequently, $10 \mu 1 \mathrm{MTT}$ $(5 \mathrm{mg} / \mathrm{ml})$ was added to cells. After $4 \mathrm{~h}$ incubation at $37^{\circ} \mathrm{C}$, the cell medium was removed completely and $100 \mu 1$ DMSO was added in cells to resolve the blue formazan crystals of live cells. The optical density of cells/well was measured at absorbance wavelength $570 \mathrm{~nm}$ using the Multiskan Spectrum Microplate Reader (Tecan Group, Ltd., Mannedorf, Switzerland). Finally, cell viability in the different treated groups $(0,2,4,6,8,10,12$ and $14 \mu \mathrm{M}$ solamargine) was calculated as a proportion, using the formula: Cell viability $(\%)=\left(\mathrm{OD}_{570 \mathrm{~nm}}-\mathrm{OD}_{630 \mathrm{~nm}}\right)_{\text {treated }} /\left(\mathrm{OD}_{570}\right.$ $\left.\mathrm{nm}^{-}-\mathrm{OD}_{630 \mathrm{~nm}}\right)_{\text {untreated }} \mathrm{x} 100 \%$.

Flow cytometry for detecting apoptosis. QBC939 cells, in the period of logarithmic phase, were seeded in 6-well plates ( $3 \times 10^{5}$ cells/well, in $2 \mathrm{ml}$ RPMI-1640 medium) and cultured overnight at $37^{\circ} \mathrm{C}$ in an atmosphere containing $5 \% \mathrm{CO}_{2}$. Cells were allowed to culture to $70 \%$ confluence/well and were treated with solamargine at the indicated concentration $(0,2$, $4,6,8$ and $10 \mu \mathrm{M})$ for $24 \mathrm{~h}$ at $37^{\circ} \mathrm{C}$. Cells were digested using $0.25 \%$ Trypsin-EDTA at $37^{\circ} \mathrm{C}$, washed with PBS and resuspended in $100 \mu \mathrm{l} 1 \mathrm{X}$ Binding Buffer (included in the Annexin V-fluorescein isothiocyanate (FITC) apoptosis detection kit). Cells in each group $(0,2,4,6,8$ and $10 \mu \mathrm{M})$ were stained with $5 \mu \mathrm{l}$ propidium iodide (PI) and $5 \mu \mathrm{l}$ Annexin V-FITC, according to the Annexin V-fluorescein isothiocyanate (FITC) apoptosis detection kit (BD Biosciences, Franklin Lakes, NJ, USA) protocol and incubated for $15 \mathrm{~min}$ at room temperature in the dark. An aliquot of $400 \mu 11 \mathrm{X}$ Binding Buffer was added and the apoptosis of QBC939 cells was detected by Guava easyCyte 6-2L flow cytometer (Merck KGaA, Darmstadt, Germany) and analyzed by the GuavaSoft software (version 2.7; Merck KGaA, Darmstadt, Germany).

Detecting the mitochondrial membrane potential by JC-1 staining buffer. QBC939 cells in the mid-log phase were seeded in 12 -well plates ( $2 \times 10^{5}$ cells/well in $1 \mathrm{ml}$ RPMI 1640 medium) and cultured overnight at $37^{\circ} \mathrm{C}$. Cells were treated with solamargine at $2,4,6,8$ and $10 \mu \mathrm{M}$ for $24 \mathrm{~h}$ at $37^{\circ} \mathrm{C}$. The treated cells were washed with PBS two times and digested with $0.25 \%$ Trypsin-EDTA. Carbonyl cyanide 3-chlorophenylhydrazone (from the mitochondrial membrane potential assay kit) was added into the positive control well and incubated at $37^{\circ} \mathrm{C}$ for $20 \mathrm{~min}$. A total of $1 \mathrm{ml} \mathrm{JC}-1$ staining buffer was added to the wells and incubated for $20 \mathrm{~min}$ at $37^{\circ} \mathrm{C}$ in the dark. The supernatant was removed at $600 \mathrm{x}$ g for $3 \mathrm{~min}$ at room temperature. Cells were washed with JC-1 washing buffer (1X) two times and then suspended in washing buffer. Flow cytometry was conducted to detect JC-1 fluorescence and analyze the change in mitochondrial membrane potential (MMP) in QBC939 cells.

$R T$-qPCR. QBC939 cells in the mid-log phase were seeded in 6 -well plates $\left(3 \times 10^{5}\right.$ cells/well in $2 \mathrm{ml}$ RPMI 1640 medium) and cultured overnight at $37^{\circ} \mathrm{C}$. Cells were allowed to culture to $70 \%$ confluence and were treated with $0,2,4,6,8$ and $10 \mu \mathrm{M}$ 
Table I. Primers used for quantitative polymerase chain reaction.

Sequence (5'-3')

\begin{tabular}{llc}
\cline { 2 - 3 } Name & \multicolumn{1}{c}{ Forward } & \multicolumn{1}{c}{ Reverse } \\
\hline GAPDH & GCAAATTCCATGGCACCGTC & GACTCCACGACGTACTCAGC \\
Bax & GAACCATCATGGGCTGGACA & GCGTCCCAAAGTAGGAGAGG \\
Bcl-2 & GAACTGGGGGAGGATTGTGG & CCGTACAGTTCCACAAAGGC \\
XIAP & TGGCAGATTATGAAGCACGGA & GGTCTTCACTGGGCTTCCAA \\
Bcl-xL & ACTCTTCCGGGATGGGGTAA & ACAAAAGTATCCCAGCCGCC \\
\hline
\end{tabular}

Bcl-2, B-cell lymphoma-2; xL, extra large; Bax, Bcl-2-associated X protein; XIAP, X-linked inhibitor of apoptosis protein.

solamargine for $24 \mathrm{~h}$ at $37^{\circ} \mathrm{C}$. The treated cells were washed with PBS twice and total RNA in cells was extracted using the TRIzol reagent (Invitrogen; Thermo Fisher Scientific, Inc.). According to the manufacturer's protocol of the first cDNA synthesis kit for RT-qPCR, mRNA was reverse transcribed to cDNA by using the reaction system (Reaction Mix $4 \mu$, Maxima Enzyme Mix $2 \mu 1$, Template RNA $500 \mathrm{ng}$, and add nuclease-free Water to $20 \mu \mathrm{l})$ and the reaction procedure $\left(25^{\circ} \mathrm{C}\right.$ for $10 \mathrm{~min}$, $50^{\circ} \mathrm{C}$ for $15 \mathrm{~min}, 85^{\circ} \mathrm{C}$ for $5 \mathrm{~min}$ ). The reaction system included (Maxima SYBR Green/ROX qPCR Master Mix (Thermo Fisher Scientific, Inc.) $10 \mu \mathrm{l}$, forward primer $0.3 \mu \mathrm{M}$, reverse primer $0.3 \mu \mathrm{M}$, Template DNA $300 \mathrm{ng}$, and nuclease-free water until a final volume of $25 \mu \mathrm{l}$ ), the thermocycling conditions were $95^{\circ} \mathrm{C}$ for $10 \mathrm{~min}, 1 \mathrm{cycle} ; 95^{\circ} \mathrm{C}$ for $15 \mathrm{sec}, 60^{\circ} \mathrm{C}$ for $30 \mathrm{sec}$ and $72^{\circ} \mathrm{C}$ for $30 \mathrm{sec}, 40$ cycles of qPCR, the genes (Bax, Bcl-2, Bcl-xL, XIAP) mRNA relative expression were detected by fluorescence quantitative PCR equipment (Applied Biosystems, Thermo Fisher Scientific, Inc.) and analyzed by the $2^{-\Delta \Delta C a}$ method. GAPDH mRNA expression was used as the control. Primers used in the experiments were synthesized by GenScript Biotech (Nanjing, China) and are listed in Table I.

Western blot. QBC939 cells in the period of logarithmic phase were seeded in 6-well plates $\left(3 \times 10^{5}\right.$ cells/well in $2 \mathrm{ml}$ RPMI 1640 medium) and cultured overnight at $37^{\circ} \mathrm{C}$ in an atmosphere containing $5 \% \mathrm{CO}_{2}$. Cells were allowed to culture to $70 \%$ confluence/well and were treated with solamargine at the indicated concentration $(0,2,4,6,8$ and $10 \mu \mathrm{M})$ for $24 \mathrm{~h}$ at $37^{\circ} \mathrm{C}$. Cells were washed with ice cold PBS three times and digested using RIPA lysis buffer with protease phosphatase inhibitor cocktail (Thermo Scientific, Inc., Waltham, MA, USA) on ice for $5 \mathrm{~min}$. Cell lysates were selected and centrifuged $\left(12,000 \mathrm{x} \mathrm{g}, 10 \mathrm{~min}, 4^{\circ} \mathrm{C}\right)$ to remove the cell debris. Total protein concentration was determined using the BCA Protein Assay kit (Beyotime Institute of Biotechnology) and detected using the Multiskan Spectrum Microplate Reader. Cell lysates are mixed with $2 \mathrm{X}$ Sample Buffer and heated in water at $100^{\circ} \mathrm{C}$ for $5 \mathrm{~min}$. Prepared protein ( $\sim 30 \mu \mathrm{g}$ per lane) was separated using SDS-PAGE (12\% gels) and transferred onto polyvinylidene difluoride membranes (Merck KGaA). After the proteins were transferred to the PVDF membrane, protein was blocked using Tris-Buffered Saline (TBS) containing 5\% non-fat milk for $1 \mathrm{~h}$ at room temperature and incubated with primary antibody (Bax, Bcl-2, caspase3, caspase7, XIAP, PARP and $\beta$-actin, all antibodies come from Cell Signaling Technology Incorporation,
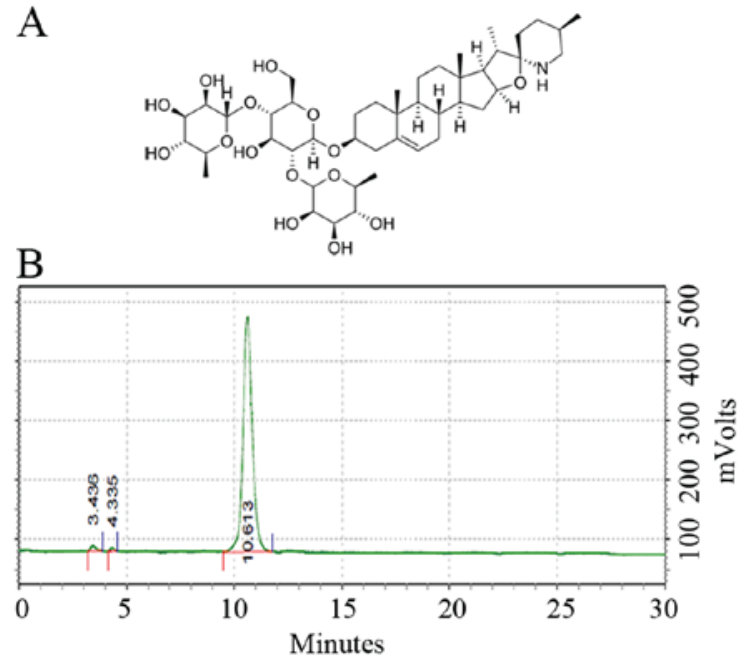

Figure 1. Solamargine. (A) Structural formula of solamargine (molecular formula, $\mathrm{C}_{45} \mathrm{H}_{73} \mathrm{NO}_{15}$; molecular weight, 868.06; CAS no. 14197-65-0). (B) Solamargine purity, determined using high-performance liquid chromatography.

antibodies were diluted by 1:1,000) overnight at $4^{\circ} \mathrm{C}$. The PVDF membrane was washed with TBS- $0.1 \%$ Tween 20 and incubated with the Dylight 800-labeled goat anti-rabbit immunoglobulin $\mathrm{G}(\mathrm{H}+\mathrm{L})$ fluorescence antibody (dilution, 1:10,000; catalog no. 072-07-15-06, KPL, Inc., Gaithersburg, MD, USA) for $1 \mathrm{~h}$ at room temperature. The blot membrane was exposed and scanned by the Odyssey infrared imaging system (LI-COR Biosciences, Lincoln, NE, USA).

Statistical analysis. Data are expressed as the mean \pm standard deviation of 3 independent experiments. The difference between different groups was analyzed using a one-way analysis of variance (with Tukey's post-hoc test) or a Student's t-test. $\mathrm{P}<0.05$ was considered to indicate a statistically significant difference. The data were analyzed using SPSS software (version 16.0; SPSS, Inc., Chicago, IL, USA) and graphs were plotted using GraphPad Prism software (version 5; GraphPad Software, Inc., La Jolla, CA, USA).

\section{Results}

Solamargine inhibits the viability and alters the morphology of cholangiocarcinoma QBC939 cells. To determine the 
A

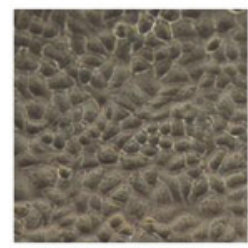

0

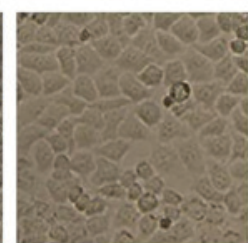

2

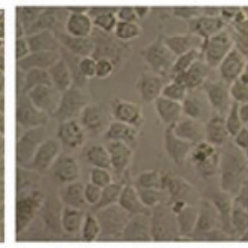

4

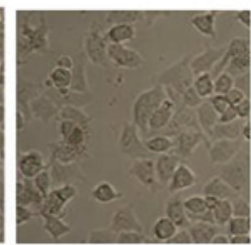

6

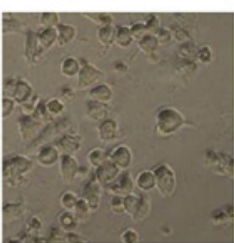

8

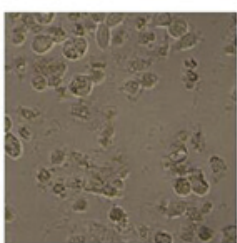

10

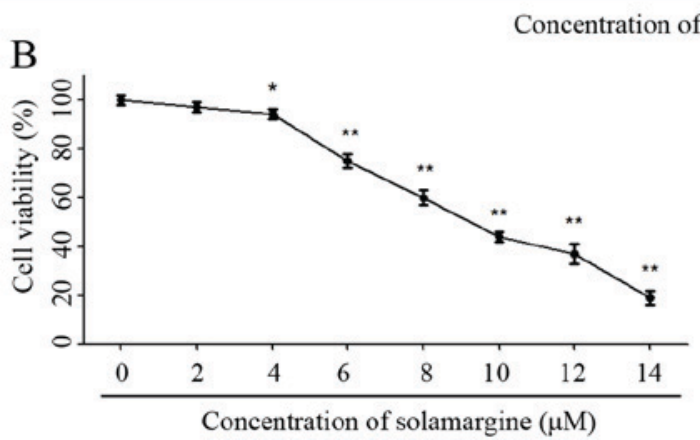

Figure 2. Effect of solamargine on cell viability and morphology of cholangiocarcinoma QBC939 cells. (A) The morphology of QBC939 cells, following treatment with different concentrations of solamargine for $24 \mathrm{~h}$, observed using light microscopy (magnification, x200). (B) QBC939 cells were treated with different concentrations of solamargine for $24 \mathrm{~h}$ and viability was determined using an MTT assay. ${ }^{*} \mathrm{P}<0.05,{ }^{* *} \mathrm{P}<0.01 \mathrm{vs}$. control.

A
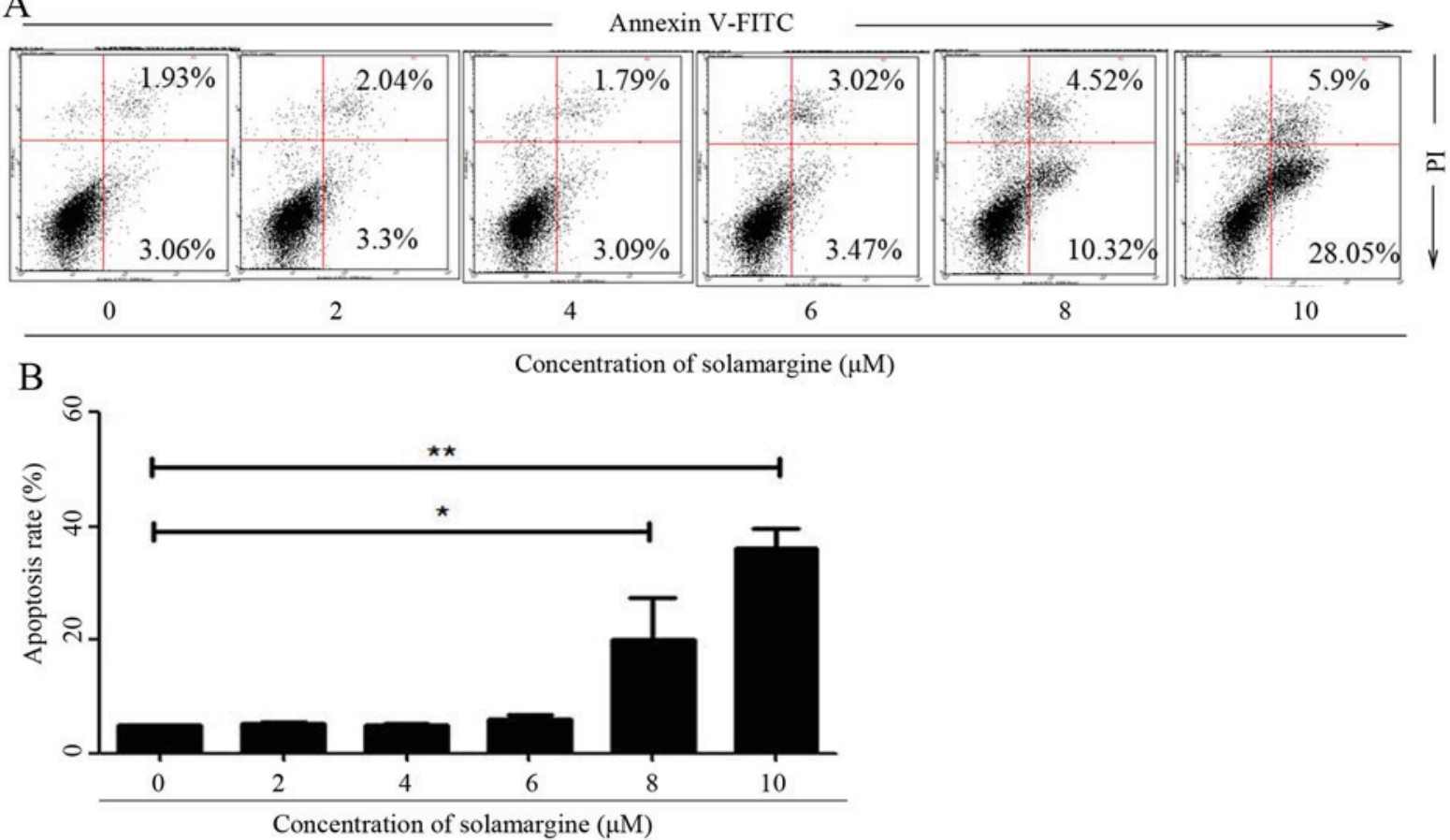

Figure 3. Cholangiocarcinoma QBC939 cells apoptosis induced by solamargine. (A) Apoptosis of QBC939 cells induced by treatment with different concentrations of solamargine for $24 \mathrm{~h}$, stained using Annexin V-FITC/PI and determined using flow cytometry. (B) Statistical data of QBC939 cell apoptosis. Apoptosis rate contains the early apoptosis rate and late apoptosis rate. ${ }^{*} \mathrm{P}<0.05,{ }^{* *} \mathrm{P}<0.01$. FITC, fluorescein isothiocyanate; $\mathrm{PI}$, propidium iodide.

precision of experiment and the quality of solamargine, HPLC was used to determine the purity of solamargine. The results revealed that the purity of solamargine was $>98 \%$ (Fig. 1B).

The effect of solamargine on QBC939 cells was observed using light microscopy (magnification, x200) at first. As the concentration of solamargine increased $(0,2,4,6,8$ and $10 \mu \mathrm{M})$, the morphology of cholangiocarcinoma cells changed markedly at $24 \mathrm{~h}$. Solamargine may cause shrinkage, irregularity and inhibit the viability of cells (Fig. 2A). The viability of human cholangiocarcinoma QBC939 cells was analyzed using an MTT assay after $24 \mathrm{~h}$ treatment with solamargine $(0,2,4,6,8,10,12 \mu \mathrm{M})$. Solamargine treatment significantly inhibited the viability of QBC939 cells in dose-dependent manner (concentration, $>4 \mu \mathrm{M}$; Fig. $2 \mathrm{~B}$ ). The half-maximal 
A
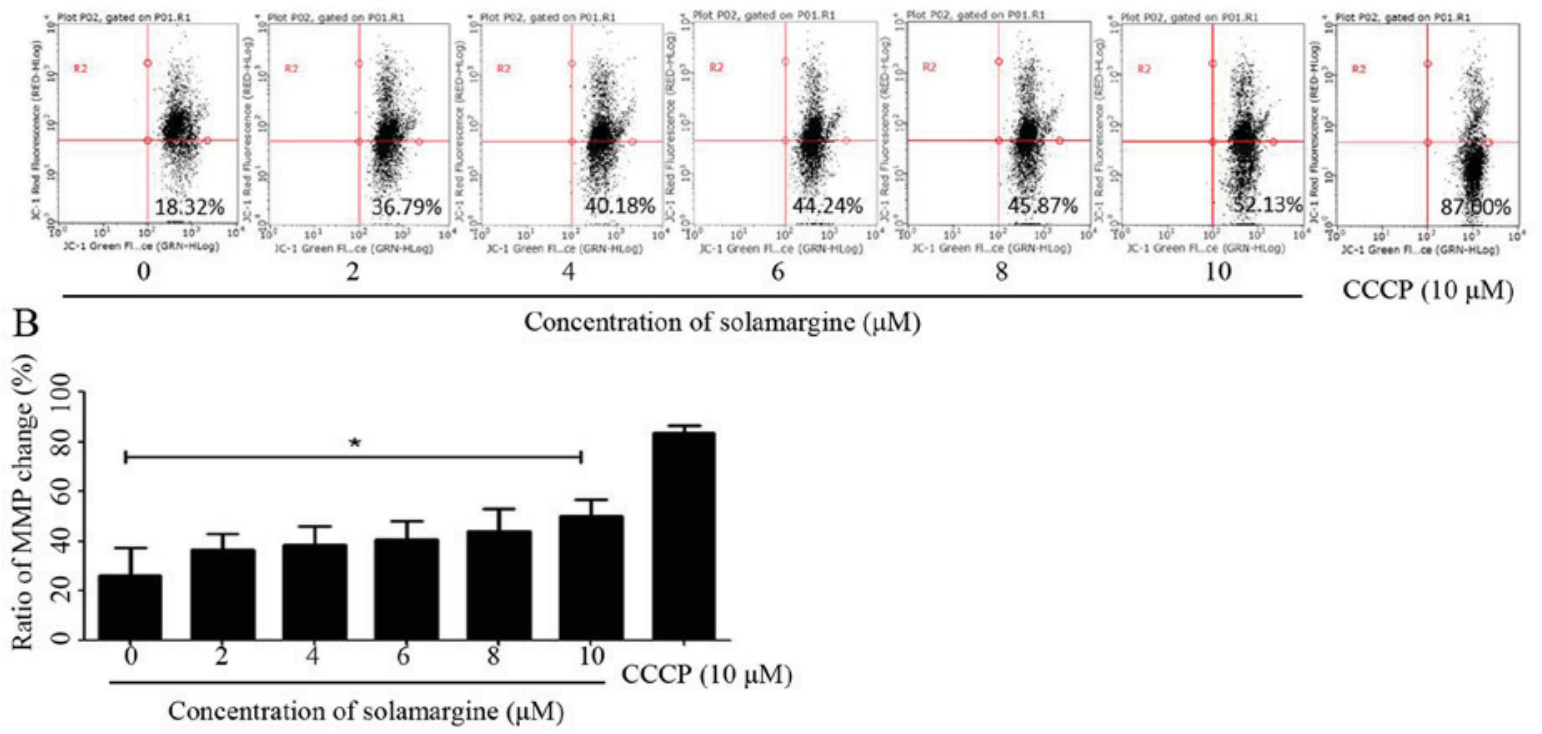

Figure 4. Alteration in MMP induced by solamargine in QBC939 cells. (A) Alteration in MMP of QBC939 cells induced by treatment with different concentrations of solamargine for $24 \mathrm{~h}$, stained with JC-1 buffer and determined using flow cytometry. Cells treated with $10 \mu \mathrm{M}$ CCCP were used as a positive control. (B) Statistical data of change rate of mitochondrial membrane potential in QBC939 cells. * $\mathrm{P}<0.05$. CCCP, carbonyl cyanide 3-chlorophenylhydrazone; MMP, mitochondrial membrane potential.

inhibitory concentration $\left(\mathrm{IC}_{50}\right)$ value $(9.81 \mu \mathrm{M})$ of solamargine on QBC939 cells is analyzed by SPSS software.

Solamargine induces apoptosis of cholangiocarcinoma QBC939 cells. To validate whether solamargine inhibits the viability of QBC939 cells by inducing apoptosis, the apoptosis of QBC939 cells, after $24 \mathrm{~h}$ treatment with different concentrations of solamargine $(0,2,4,6,8$ and $10 \mu \mathrm{M})$, was determined using flow cytometry. After QBC939 cells were digested and harvested, cells were stained with Annexin V-FITC and PI. Cells positive for Annexin V-FITC only represented early apoptotic cells, whereas cells positive for Annexin V-FITC and PI represented late apoptotic cells. Flow cytometric analysis revealed that solamargine induced apoptosis of cholangiocarcinoma QBC939 cells significantly in a dose-dependent manner. Solamargine significantly induced apoptosis at $>6 \mu \mathrm{M}$ and could primarily induced early apoptosis (Fig. 3).

Solamargine alters the mitochondrial membrane potential in QBC939 cells. The present study identified that solamargine may induce the pro-apoptosis of human cholangiocarcinoma cells significantly. The alteration in mitochondrial membrane potential may lead to early apoptosis (14). Therefore, in the present study, QBC939 cells were treated with different concentrations of solamargine $(0,2,4,6,8$ and $10 \mu \mathrm{M})$ for $24 \mathrm{~h}$ and stained with JC-1 staining buffer for $20 \mathrm{~min}$ in the dark. Subsequently, the MMP of QBC939 cells was determined using flow cytometry. As presented in Fig. 4A, compared with the untreated group, the results demonstrated an increase in green fluorescence and a decrease in red fluorescence, which indicated that solamargine caused a depolarized MMP in QBC939 cells (Fig. 4B).

Solamargine alters the expression and activation of apoptosis-associated proteins in QBC939 cells. Based on the above results, the molecular mechanism of apoptosis effect of solamargine on human cholangiocarcinoma QBC939 cells was subsequently investigated. The present study assessed the expression of apoptosis-associated proteins in QBC939 cells, after $24 \mathrm{~h}$ treatment with different concentrations of solamargine $(0,2,4,6,8$ and $10 \mu \mathrm{M})$, using RT-qPCR and western blot analysis. The total RNA of QBC939 cells was extracted using TRIzol reagent and the mRNA was transcribed into cDNA. The relative expression of Bax, Bcl-2, Bcl-extra-large (Bcl-xL) and XIAP mRNA was determined using RT-qPCR (using GAPDH as the reference gene). The results revealed that solamargine significantly inhibited Bcl-2 and XIAP mRNA levels but significantly increased the mRNA level of Bax (Fig. 5A). The total protein of QBC939 cells was extracted using RIPA cell lysis buffer, after $24 \mathrm{~h}$ incubation, and quantitated using the BCA protein assay kit. The protein levels of Bax, Bcl-2, caspase 3 , cleaved-caspase 3, caspase 7, XIAP, PARP and cleaved PARP were determined using western blot analysis. As presented in Fig. 5B, solamargine increased the protein expression of Bax, caspase 3, cleaved-caspase 3, caspase 7 and cleaved PARP, but decreased the protein expression of Bcl-2, XIAP and PARP. Therefore, solamargine may influence apoptosis-associated proteins to inhibit the viability of QBC939 cells.

\section{Discussion}

Cholangiocarcinoma is a type of aggressive and refractory malignancy, and characterized by late diagnosis and poor outcomes; therefore, identifying novel therapeutic drugs is required (2,17). Solamargine, a type of alkaloid derived from $S$. nigrum, may inhibit the proliferation of and induce apoptosis in multiple types of tumor cell lines (including human breast cancer, lung cancer and hepatocellular carcinoma cell lines) (7-13). The present study explored the therapeutic effect of solamargine on human cholangiocarcinoma QBC939 cells preliminarily. The present study revealed that alkaloid 
A
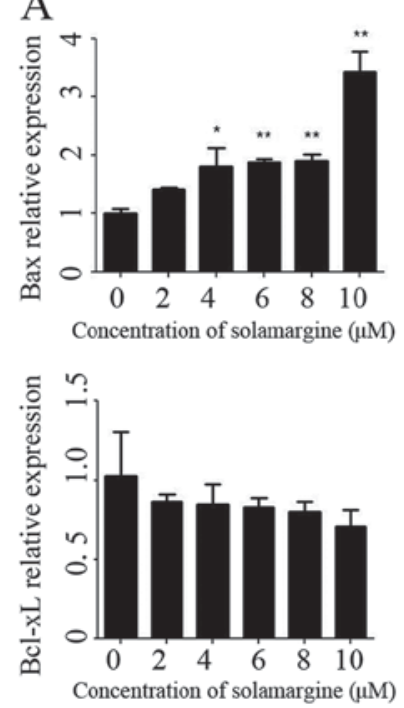
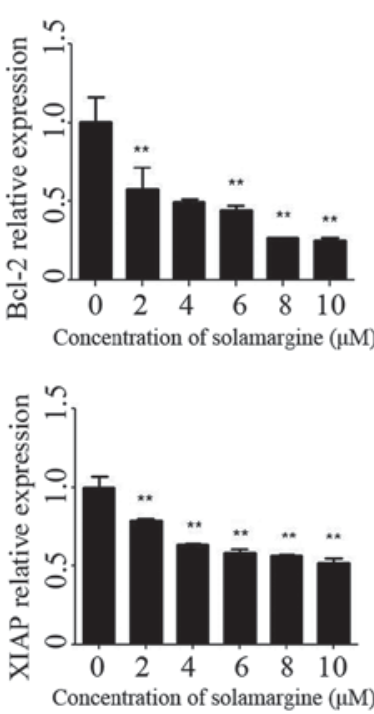

B

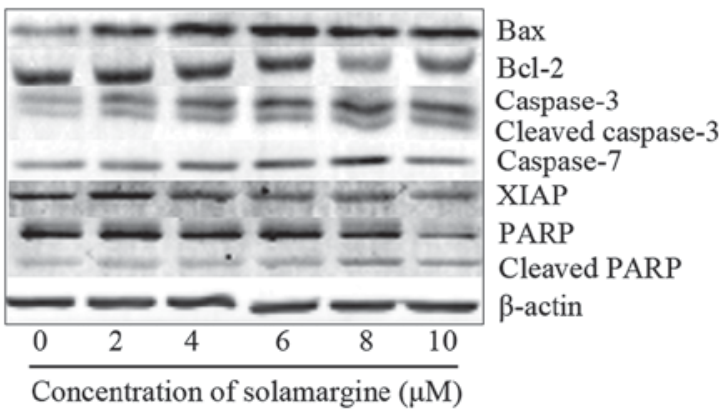

Figure 5. Effect of solamargine on the expression and activation of apoptosis-associated proteins. (A) The expression of Bax increased with solamargine treatment; whereas the expression of Bcl-2, Bcl-xL and XIAP decreased with solamargine treatment. Determined using quantitative polymerase chain reaction, with GAPDH used as the internal control. (B) Western blot analysis indicated that solamargine treatment increased expression of Bax, caspase-3, cleaved caspase-3, caspase-7 and cleaved PARP but decreased the expression of Bcl-2, XIAP and PARP. $\beta$-actin was used as the internal control. ${ }^{*} \mathrm{P}<0.05,{ }^{* *} \mathrm{P}<0.01$ vs. control. Bcl-2, B-cell lymphoma-2; Bcl-xL, Bcl-extra-large; Bax, Bcl-2-associated X protein; XIAP, X-linked inhibitor of apoptosis protein; PARP, poly ADP ribose polymerase.

solamargine treatment on QBC939 cells can result in the emergence of cell shrinkage, irregularity and apoptotic bodies, which were observed by using a light microscope. An MTT assay revealed that solamargine inhibited the cell viability of QBC939 cells in the dose-dependent manner and the value of $\mathrm{IC}_{50}$ was $9.81 \mu \mathrm{M}$. The results of the present study suggested that solamargine may be a chemotherapeutic drug for the treatment of human cholangiocarcinoma. Therefore, future studies may focus on exploring how solamargine inhibits the viability of QBC939 cells. Apoptosis is a type of programmed cell death and could serve as an important antitumor target $(15,18)$. Induction of apoptosis in the tumor microenvironment may inhibit excessive cell proliferation and is an effective therapeutic strategy against cancer (19). The present study demonstrated that solamargine induced apoptosis of QBC939 cells significantly in a dose-dependent manner, as determined using flow cytometry. A previous study revealed that the alteration in MMP is an early event of pro-apoptosis and may result in the release of cytochrome $c$ in mitochondria, which may induce the activation of caspase 9 (14). The alterations in MMP induced by solamargine, determined using flow cytometry in the present study, demonstrated that solamargine could change MMP in QBC939 cells. Apoptosis is associated with multiple pro-apoptotic proteins (including Bax and caspase 3) and anti-apoptotic proteins (including Bcl-2 and XIAP) and is determined by the ratio of pro-to anti-apoptotic proteins (20). To evaluate the underlying molecular mechanism of solamargine-induced apoptosis, the alterations in apoptosis-associated gene and protein expression were detected using RT-qPCR and western blot analysis. The results indicated that solamargine increased the expression of Bax, caspase 3, cleaved caspase 3, caspase 7 and cleaved PARP and decreased the expression of $\mathrm{Bcl}-2, \mathrm{Bcl}-\mathrm{xL}$, XIAP and PARP. Therefore, solamargine may be an effective chemotherapeutic agent against cholangiocarcinoma by inducing apoptosis.
The results of the present study indicated that solamargine may induce apoptosis significantly in human cholangiocarcinoma QBC939 cells via the MMP pathway. Solamargine is the one member of natural compounds (luteolin, matrine, berberine and so on) against human cholangiocarcinoma $(21,22)$, and the results of the present study suggested that solamargine may be an effective drug candidate against cholangiocarcinoma. Since the present study only included in vivo experiments, additional in vitro assays are required to validate whether solamargine may be a therapeutic agent for the treatment of cholangiocarcinoma.

\section{Acknowledgements}

Not applicable.

\section{Funding}

The present study was supported by the Research Innovation Program for Academic Degree Postgraduate of Jiangsu Province General University (grant no. 2014965) and the Key Project supported by the Medical Science and Technology Development Foundation, Nanjing Department of Health (grant no. YKK14176).

\section{Availability of data and materials}

All data generated or analyzed during this study are included in this published article.

\section{Author's contributions}

Cell culture and apoptosis detection were conducted by $\mathrm{XZ}$ and ZY. Western blotting and RT-qPCR were performed by TX and ZA. HPLC was analyzed by MH. Experimental data 
were analyzed by WC and XW. FZ and ZY were the major contributors to study design and wrote the manuscript. All authors read and approved the final manuscript.

\section{Ethics approval and consent to participate}

Not applicable.

\section{Consent for publication}

Not applicable.

\section{Competing interests}

The authors declare that they have no competing interests.

\section{References}

1. Plentz RR and Malek NP: Clinical presentation, risk factors and staging systems of cholangiocarcinoma. Best Pract Res Clin Gastroenterol 29: 245-252, 2015.

2. Bergquist A and von Seth E: Epidemiology of cholangiocarcinoma. Best Pract Res Clin Gastroenterol 29: 221-232, 2015.

3. Rizvi S and Gores GJ: Pathogenesis, diagnosis, and management of cholangiocarcinoma. Gastroenterology 145: 1215-1229, 2013.

4. Rizvi S, Borad MJ, Patel T and Gores GJ: Cholangiocarcinoma: Molecular pathways and therapeutic opportunities. Semin Liver Dis 34: 456-464, 2014.

5. Zhu AX: Future directions in the treatment of cholangiocarcinoma. Best Pract Res Clin Gastroenterol 29: 355-361, 2015.

6. Ding X, Zhu F, Yang Y and Li M: Purification, antitumor activity in vitro of steroidal glycoalkaloids from black nightshade (Solanum nigrum L.). Food Chem 141: 1181-1186, 2013.

7. Munari CC, de Oliveira PF, Campos JC, Martins Sde P, Da Costa JC, Bastos JK and Tavares DC: Antiproliferative activity of Solanum lycocarpum alkaloidic extract and their constituents, solamargine and solasonine, in tumor cell lines. J Nat Med 68: 236-241, 2014.

8. Ding X, Zhu FS, Li M and Gao SG: Induction of apoptosis in human hepatoma SMMC-7721 cells by solamargine from Solanum nigrum L. J Ethnopharmacol 139: 599-604, 2012.

9. Sani IK, Marashi SH and Kalalinia F: Solamargine inhibits migration and invasion of human hepatocellular carcinoma cells through down-regulation of matrix metalloproteinases 2 and 9 expression and activity. Toxicol In Vitro 29: 893-900, 2015.
10. Xie X, Zhu H, Yang H, Huang W, Wu Y, Wang Y, Luo Y, Wang D and Shao G: Solamargine triggers hepatoma cell death through apoptosis. Oncol Lett 10: 168-174, 2015.

11. Shiu LY, Chang LC, Liang CH, Huang YS, Sheu HM and Kuo KW: Solamargine induces apoptosis and sensitizes breast cancer cells to cisplatin. Food Chem Toxicol 45: 2155-2164, 2007.

12. Chen Y, Tang Q, Wu J, Zheng F, Yang L and Hann SS: Inactivation of PI3-K/Akt and reduction of SP1 and p65 expression increase the effect of solamargine on suppressing EP4 expression in human lung cancer cells. J Exp Clin Cancer Res 34: 154, 2015.

13. Liang CH, Shiu LY, Chang LC, Sheu HM, Tsai EM and Kuo KW: Solamargine enhances HER2 expression and increases the susceptibility of human lung cancer H661 and H69 cells to trastuzumab and epirubicin. Chem Res Toxicol 21: 393-399, 2008.

14. Koff JL, Ramachandiran S and Bernal-Mizrachi L: A time to kill: Targeting apoptosis in cancer. Int J Mol Sci 16: 2942-2955, 2015.

15. Fulda S: Targeting apoptosis for anticancer therapy. Semin Cancer Biol 31: 84-88, 2015.

16. Su Z, Yang Z, Xu Y, Chen Y and Yu Q: Apoptosis, autophagy, necroptosis, and cancer metastasis. Mol Cancer 14: 48, 2015.

17. Razumilava N and Gores GJ: Cholangiocarcinoma. Lancet 383: 2168-2179, 2014

18. Fulda S: Targeting extrinsic apoptosis in cancer: Challenges and opportunities. Semin Cell Dev Biol 39: 20-25, 2015.

19. Lopez J and Tait SW: Mitochondrial apoptosis: Killing cancer using the enemy within. Br J Cancer 112: 957-962, 2015.

20. Goldar S, Khaniani MS, Derakhshan SM and Baradaran B: Molecular mechanisms of apoptosis and roles in cancer development and treatment. Asian Pac J Cancer Prev 16: 2129-2144, 2015.

21. Aneknan P, Kukongviriyapan V, Prawan A, Kongpetch S, Sripa B and Senggunprai L: Luteolin arrests cell cycling, induces apoptosis and inhibits the JAK/STAT3 pathway in human cholangiocarcinoma cells. Asian Pac J Cancer Prev 15: 5071-5076, 2014.

22. Yang N, Han F, Cui H, Huang J, Wang T, Zhou Y and Zhou J: Matrine suppresses proliferation and induces apoptosis in human cholangiocarcinoma cells through suppression of JAK2/STAT3 signaling. Pharmacol Rep 67: 388-393, 2015. 